\title{
Web-3DPredictor: a web interface for high-resolution prediction of genome architecture
}

\author{
Emil Valeev \\ ICG SB RAS, Novosibirsk, Russia \\ emil@bionet.nsc.ru
}

\author{
Polina Belokopytova \\ ICG SB RAS, Novosibirsk, Russia \\ belokopytova@bionet.nsc.ru
}

\author{
Veniamin Fishman \\ ICG SB RAS, Novosibirsk, Russia \\ NSU, Novosibirsk, Russia \\ minja@bionet.nsc.ru
}

\begin{abstract}
Alterations of spatial contacts of chromatin may lead to developmental disorders and cancer. We have recently shown that 3-dimensional genomic contacts could be predicted in normal and rearranged genomes using epigenetic information. Here we describe an extension of this algorithm, characterized by more accurate and higher-resolution predictions, and its web implementation which allows researchers and clinical doctors to generate patient-specific predictions of genome architecture.
\end{abstract}

Index Terms - Hi-C, chromatin organization, CTCF, RNAseq, epigenetic mechanisms

\section{Motivation and aim}

\section{Motivation}

It was recently shown that chromatin contacts could be predicted using widely-available epigenetic information and machine-learning methods [1]. These predictions could be used to dissect molecular mechanisms which connect chromosomal rearrangements with human diseases, and thus could provide useful resource for clinical genetics. However, the majority of the developed predictive algorithms are not available as a stand-alone or online software; therefore, their usage in clinics is limited. Moreover, the resolution of these algorithms is often low, thus estimation of the clinical significance of changes in 3D-genome architecture is challenging.

Aim

We aimed to extend our recently developed computational tool, 3DPredictor, to predict chromatin contacts at high resolution $(1 \mathrm{~kb})$. Moreover, we aimed to develop a web application that allows to predict genome organization in normal and rearranged genomes, for researchers and clinical doctors who are not experienced in bioinformatics.

\section{Methods}

We used ML-based algorithm detailed in [1] to train models and predict chromatin 3D organization. Training datasets which high-resolution prediction required were taken from [2]. For web service development we used the following tools:
- Front-end: PureCSS framework, PHP

- Back-end:

- $\quad$ Pipeline: Bash script

- $\quad$ Preprocessing CTCF data: gimmemotifs [3]

- $\quad$ Preprocessing RNA-seq data: Python3 script

- Hi-C map: Juicer Tools [4]

\section{Results}

We showed that prediction can be significantly improved by training algorithm on higher resolution data. Also, we made a web service which performs prediction of chromatin 3Dstructure for a region of interest. Trained models for $H$. sapiens and M. musculus at $1-$ and $5-\mathrm{kb}$ resolutions are provided. Web3DPredictor is hosted on the ICG server ${ }^{1}$. Both source code and trained models could be obtained on GitHub to deploy local server elsewhere.

\section{AKNOWLEDGEMENT}

Supported by the RFBR (18-29-13021).

\section{REFERENCES}

[1] Polina S. Belokopytova, Miroslav A. Nuriddinov, Evgeniy A. Mozheiko, Daniil Fishman and Veniamin Fishman, "Quantitative prediction of enhancer-promoter interactions", Genome Research, 2019. doi: 10.1101/gr.249367.119

[2] Nils Krietenstein, Sameer Abraham, Sergey V. Venev, Nezar Abdennur, Johan Gibcus, Tsung-Han S. Hsieh, Krishna Mohan Parsi, Liyan Yang, Rene Maehr, Leonid A. Mirny, Job Dekker, Oliver J. Rando, "Ultra-' structural details of mammalian chromosome architecture", 2019. doi: 10.1101/639922

[3] Niklas Bruse, Simon J. van Heeringen, "GimmeMotifs: an analysis framework for transcription factor motif analysis", 2018. doi: $10.1101 / 474403$

[4] Neva C. Durand, Muhammad S. Shamim, Ido Machol, Suhas S. P. Rao, Miriam H. Huntley, Eric S. Lander, and Erez Lieberman Aiden, "Juicer provides a one-click system for analyzing loop-resolution $\mathrm{Hi}-\mathrm{C}$ experiments", Cell Systems 3(1), $2016 . \quad$ doi: 10.1016/j.cels.2016.07.002 